\title{
Capturing and Sharing Memories in a Virtual World
}

\author{
Carman Neustaedter and Elena Fedorovskaya \\ Kodak Research Labs \\ 1999 Lake Avenue, Rochester, NY, USA \\ [firstname.lastname]@kodak.com
}

\begin{abstract}
Virtual worlds (VWs) such as Second Life ${ }^{\circledR}$ (SL) contain a rich social culture where people engage in a multitude of experiences much like real life. With this comes the need to capture and share memories with others. To understand what tools people use to accomplish this and what limitations they may face, we conducted interviews with participants in SL. Our results identify two clusters of users-Casuals and Lifers-who differed in the ways in which they captured and shared memories. Here we describe the use of photos, landmarks, friend lists, and conversation logs. We also show how a lack of real life physical and social constraints in the VW affects user routines, and, in some cases, how it does not. This suggests design directions for memory tools in the VW and also real life that break the bounds of current everyday practice.
\end{abstract}

\section{Author Keywords}

Virtual worlds, memories, photo sharing, Second Life.

\section{ACM Classification Keywords}

H5.3. [Group and Organization Interfaces]: Synchronous.

\section{INTRODUCTION}

Virtual worlds (VWs) are computer-based environments that are simulated, shared, and persistent [3]. Over the years, they have evolved from adventure-style games in the form of textual-based multi-user dungeons (MUDs) [3,7] to sophisticated 3D environments where users navigate in real time through virtual representations, often in the form of avatars [3]. Regardless of the technical sophistication, VWs have always been places of social interaction [3]. Their use has also evolved to include collaboration [10] and social networking [4] in addition to gaming [9].

Our interest is in VWs found in Multi-User Virtual Environments (MUVEs) that focus less on gaming and more on social activities (e.g., Active Worlds, Kaneva, Second Life). People enter these VWs with the typical

Permission to make digital or hard copies of all or part of this work for personal or classroom use is granted without fee provided that copies are not made or distributed for profit or commercial advantage and that copies bear this notice and the full citation on the first page. To copy otherwise, or republish, to post on servers or to redistribute to lists, requires prior specific permission and/or a fee.

CHI 2009, April 3-9, 2009, Boston, MA, USA.

Copyright 2009 ACM 978-1-60558-246-7/08/04 ...\$5.00 intention of meeting and interacting with people from around the world. They engage in a variety of activities such as chatting, dancing, or traveling. They create friendships and sometimes even find love. Within these VWs, it is clear that a rich social culture is developing that is unconstrained by many real world physical and social constraints [30]. For example, people may be able to travel to a different location in a matter of seconds or masquerade as their ideal social self with little or no social inhibitions. What is not clear is how this lack of constraints engenders unique cultural implications in VWs.

Researchers have begun to address this by studying a variety of topics in MUVEs including education, identity, collaboration [4], and social activities [9]. Our focus is in understanding how and why users capture and share memories of their experiences and activities in the VW. Research has shown that VW participants actively record their experiences through blogs [29], narrative [8], and video (e.g., machinima) [17,25]. Our work builds on this research by investigating how users utilize photos along with other in-world tools to capture and share their experiences and happenings. We accomplish this through a series of interviews with participants in the VW of Second Life (SL). Our findings show that user routines vary between two types of users-Casuals and Lifers-who exhibit different degrees of permanence in the VW. We also contrast these routines with RL routines for capturing and sharing memories illustrated in the related literature.

These findings provide knowledge that researchers, designers, and practitioners can use to understand VW activities and spur creative thought for designing applications for VWs. Our findings also allow us to see what people do in an environment that is not bound by many of the physical and social constraints of RL. These behaviors can then act as a catalyst for RL technology design that may break the bounds of everyday practice.

\section{SECOND LIFE}

Second Life ${ }^{\circledR}$ is a VW launched in 2003 by Linden Lab. SL is arguably not a game: there is no overarching reward structure, levels, or points associated with play. Instead, SL is a MUVE where players can travel, meet other people, and pursue a wide range of activities much like in real life (denoted as RL in SL chats). Users enter SL and create a human-like avatar as their virtual representation (although some choose to use animal body parts). Avatars often begin 


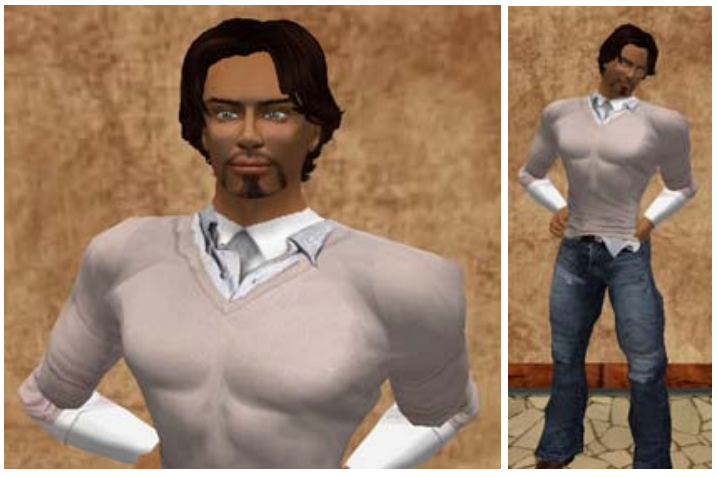

Figure 1. A Second Life ${ }^{\circledR}$ avatar.

with a fairly generic look created by modifying predefined attributes. Over time, many purchase appearance items (e.g., bodies, hair, clothes) from stores within SL to further refine one's look (Figure 1). Each avatar also has a Profile that others can view. It lists the avatar's name, creation date, and any groups to which the person belongs. There is also space to include a photo, describe oneself, list a partner, list favorite places, and describe a RL.

SL is a free-form VW in the sense that users create and construct the world themselves. Land is for sale and on it users are able to construct their own buildings and objects. To support economic activities, SL has its own currency, the Linden Dollar (L\$), which can be traded for US Dollars (as of Dec 2008, 1 USD = 260 L\$). Basic navigation in the SL "grid" involves walking, flying, and even teleporting. Communication with others is done via a chat window, which broadcasts text to nearby people or through private instant messages between individuals. Users can also enable an audio link for voice chat, although use of this is less common.

Social interaction is really the heart of the subculture that we have studied in SL. People meet others, interact, and sometimes even form close relationships. The next section outlines the study we have conducted to explore this theme and understand how people capture and share memories of their SL experiences and interactions.

\section{METHODOLOGY}

We created two avatars (one male, one female) within SL and participated in observations and interactions over a period of four months involving several hours of use per week. This included participating in a range of events, from socializing (e.g., "hanging out"), to visiting specific locations (e.g., the Eiffel Tower, the beach), to partaking in activities such as shopping, dancing, etc. These activities provided us with a firsthand account of the ways people experience SL, capture memories, and share them. We built on this knowledge by conducting semi-structured interviews with 20 SL participants. Interview questions varied, but usually began by eliciting one's basic experiences in SL like frequency of play, motivations for joining and continuing to play, favorite activities, etc. We then asked participants to describe how they captured and shared memories within SL, focusing on the use of photos and other tools found within the SL interface. Whenever possible, we had participants share their memories with us; this involved showing us photos or taking us to particular locations, along with conversing. Our interview questions iteratively evolved from participant to participant as we learned more details about the topic area. Interviews varied from twenty minutes to over an hour and sometimes we met with people multiple times to gather more information.

\section{Interview Participants}

Interviewees were recruited casually during our interactions within SL and some became friends with us (albeit, weak ties) either before or after the interviews. In certain cases, building a relationship was a prerequisite to obtaining important details about a participant's experiences. However, in most cases, people were willing to talk with us as complete strangers. We met respondents in welcome areas, shopping malls, dance clubs, cafes, and even private residences. Not everyone we talked to in SL became participants; we screened people using an initial set of questions, which varied based on the demographics we were trying to meet. Our focus was on users who could be considered part of a "friendship" culture where people aspire to meet others and create friendships. This is in contrast to niche communities with specific foci (e.g., trysts) [2]. All participants were informed that we were researchers studying the social culture of SL and willingly agreed to share their experiences and thoughts. Participants were not remunerated in any way; most, if not all, agreed to be interviewed merely for personal pleasure.

We interviewed 8 male avatars and 12 female avatars with a said age range of 20-48 years old (median of 35 years). Of course, the accuracy of this demographic information is questionable given that it is easy to fabricate one's identity in the VW. Despite this, we think that we had a reasonably diverse sample. Those participants who disclosed their RL locations to us were from a range of countries in North America and Europe. Not all were native English speakers, but this is the language we corresponded in with all. Avatars ranged from 1 to 18 months old (each avatar's profile lists its creation date); however, we learned that some people create a new avatar after a period of time. Thus, it is possible that some of our participants had experienced many more months in SL (as other avatars) than we were able to discern from their profiles. Frequency of in-world time ranged from daily to weekly visits and varied in the amount of time spent per visit from a few minutes to several hours (e.g., 8-10 hours). Thus, we had a wide range of experience and immersion.

\section{Data Collection and Analysis}

We logged all of our chat transcripts and kept field notes of our activities. We then analyzed over 11,000 lines of chat and over 100 pages of field notes using an open-coding process where important passages and quotes were marked with representative codes [28].

Our results focus on qualitative aspects of our inquiry where we present representative stories and quotes from our 
data. All avatar names refer to fictitious pseudonyms and the orthography in the presented chat logs is unaltered. Chat lines not germane to the results have been replaced with ellipses for clarity. Our study did not compare the RL routines of our participants to their SL ones. In SL, asking too many details about one's RL is often seen as taboo, at least for strangers or weak ties. To address this, we discuss our findings in relation to existing studies of RL routines (where available) and offer comparisons.

\section{TWO TYPES OF EXPERIENCES}

A good first question is: what are people doing in SL that even warrants capturing and sharing memories? Most surprising to us was that many people in SL replicate facets of RL. That is, they try to fulfill a series of needs, which include security of relationships, property, and employment (among other things) [19]. Within this sphere of activities, we identified two broad groupings of users which exhibited different virtual world experiences: Lifers, who exhibited a high degree of permanence in the VW, and Casuals, who exhibited a low degree of permanence.

In general, each group had distinctive traits surrounding users' memory capturing and sharing routines, though certain aspects overlapped. Naturally, both of the groupings are part of a continuum of VW immersion, rather than a binary choice. Users who fell into the extreme of each group differed greatly. Users who fell into the middle of the continuum exhibited properties of both groups. We did not find evidence suggesting the groupings were based on age, or duration or frequency of play. In fact, we saw some Casuals who came to SL as regularly as some Lifers. Our participants comprised 12 Lifers and 8 Casuals. Of the 12 Lifers we studied, 10 were female avatars and 2 were male avatars; 6 of 8 Casuals were male avatars. This suggests that female avatars are more often Lifers.

Other research has classified VW users ([3] summarizes) according to their goal or level of self-identification. While valuable, the categorization we have derived is more applicable for studying memory collection as it directly identifies the experiences people are capturing and sharing.

\section{LIFERS}

Lifers were SL users who were significantly immersed in the VW and had a high degree of permanence. That is, they participated in VW activities which created a need to return to the VW to fulfill certain social obligations. In general, Lifers took part in one or more of three SL activities that replicated RL: having a partner, owning/renting a residence, and/or working at a job. On the extreme, there existed SL users who did all three activities.

For example, we interviewed, Helena, a 39-year-old wife and mother of two teenage children in RL. Helena owned and occupied a house on a SL island. This meant that it was a common place for her to hang out, invite friends to, change clothes, etc. The house was fully furnished and she even had a dog named Fritz that ran to greet her. Helena had three jobs in SL: a counselor, a teacher at a SL university, and a hostess at a dance club. Throughout her year in SL, Helena had made many friends and even had a SL boyfriend that she "lived with" in her virtual home. Helena has had many experiences with her friends involving travels, dinners, and romantic dances. In addition to Helena, there were other interviewees who were significantly immersed in SL and had a high degree of permanence yet took part in only a subset of the three activities (partner, home, job).

\section{Capturing Experiences}

Many Lifers had a strong need to capture their VW experiences, given their high degree of permanence. They wanted to have ways to record what activities they did and with whom they did them. Here we found that 11 of 12 Lifers in our study used photos to capture experiences, albeit some more than others. Aside from keeping a diary or chat log (only done by three Lifers), our Lifers did not use any other techniques for capturing experiences. As such, the following sections focus heavily on the use of photos.

\section{Capturing Experiences through Photos}

The SL client currently contains a "Snapshot" tool that lets users take pictures of their SL client window (with or without the user interface controls). Users can adjust the camera angle, position, and zoom to capture from virtually anywhere and in anyway. Poses can also be pre-scripted to create a seemingly "perfect" moment. An audible "clicking" sound alerts those nearby in SL that a photo was taken. Once captured, users can email the photo to someone or save it to their hard drive (no cost). Users can also pay $10 \mathrm{~L} \$$ (less than \$0.03 USD) to save a snapshot in their SL inventory or upload a photo from their hard drive into SL. Inventories are private and others cannot see the contents.

Six of the Lifers in our study relied extensively on photos for capturing SL experiences and had large amounts of photos (several thousand). Five Lifers captured photos but had more modest amounts (in the tens to hundreds). For example, Helena, had over 2000 photos of her SL experiences. These typically were pictures of friends, locations, activities, or funny events that she wanted to remember. Helena even had pictures of her avatar as she evolved from conception until the present. Sometimes Helena would attach notes to photos to remember specific details of what was being captured. Of the photos Helena shared with us (over fifty), all contained carefully constructed poses with people.

The photo-capture routines employed by users like Helena were very similar to photo capture practices in RL. They reflect a "Kodak Culture" of photography (coined by [5]) that comprises people taking carefully constructed portraits of friends/family [5,20]. These photos represent an idealized reality that people wish to portray to others (e.g., always smiling faces), rather than realistic situations [5].

Yet we also found others who spontaneously captured photos that were less constructed. For example, Taylor, a 21-year-old nursing student in RL, had several hundred SL 


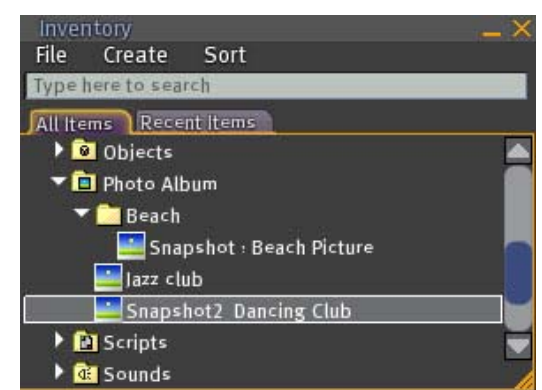

Figure 2. Photos stored in an avatar's inventory.

photos and described them as a mix of spontaneous capture and posed portraits ("sim" refers to simulation):

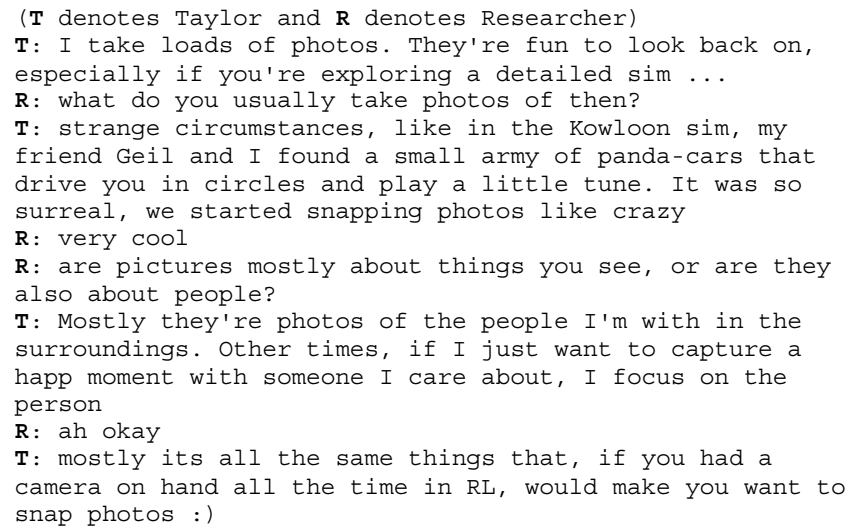

Taylor's use of photos in SL is indicative of a photo-capture culture from RL that has emerged along with ubiquitous cameras (e.g., digital cameras, mobile phones) [16]. Here everyday things (often mundane) are captured in a highly spontaneous fashion $[16,18,20]$. In this way, we see more similarities between RL and SL photo capture.

Taken together, we can see that despite having a variety of possibilities for photo capture (e.g., capture from any angle or zoom level), few people relied on them. Instead they captured photos in much the same way that people do in RL. In this case, the possibility of a VW that is unconstrained by the physical and social barriers of RL did not affect the everyday practices of its users. People simply replicated RL photo capturing behaviors in the VW.

\section{Searching (Browsing) for Photos}

The main challenge that users faced when capturing experiences through photos was their organization. This was especially the case for users like Helena and Taylor who captured large volumes of photos. In RL, searching through photos to find particular ones can be a challenge and often this activity turns into browsing [27]. The same is true in SL: to find photos people browsed through collections until they found photos of interest.

Figure 2 shows an avatar's inventory window in SL where each photo appears within a hierarchical tree-list. Under the folder "Photo Album," you can see this user has three photos, one of which is in the user-created subfolder labeled "Beach." Photos can easily be renamed to help with organization. Some SL users created elaborate folder structures, while others had a completely flat hierarchy:

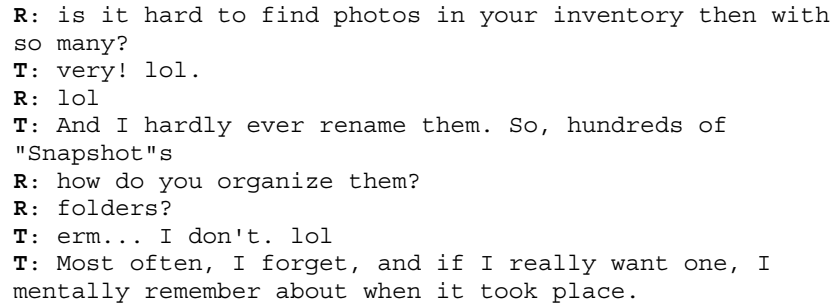

In cases when remembering was not possible, users would begin to look at photos to find the ones that interested them. Of course, SL users do have the option of storing photos on their hard drive and can then use any photo management software they choose. However, the downside is that photos are then not easily shared in SL.

\section{Sharing Experiences}

One of the main reasons that Lifers captured memories in the VW was to share their experiences with other SL users.

\section{Distributed Photo Sharing}

Distributed photo sharing was the most common form of photo sharing done by our participants and occurred when photos were directly shared between two avatars. Nearly all Lifers engaged in this act. Avatars need not be far away for distributed photo sharing to occur. In some cases, they were only a few meters apart. The distributed nature refers to the fact that photos were not shared or viewed on a common display (as would be the case with collocated sharing). Photos were actually sent to another avatar. Photos can be shared in this distributed fashion via RL email at the time of capture (as mentioned previously), or by dragging and dropping photos from one's inventory onto an avatar or a person's name in a contact list (e.g., a buddy list). Once received, a photo-viewer is opened in the recipient's client. This type of sharing was often fairly selective in terms of the recipient. For example, Alana (24 years old, unemployed in RL) only shared SL photos with her SL boyfriend because they had a close relationship. Here the photos were of shared experiences between the two.

Photos were also shared in a distributed fashion, albeit in a more public (and less directed) form. For example, currently, all users in SL have a publicly viewable profile. We found a common practice was to display a photo of one's avatar in this profile. Thus, users publicly shared photos with other avatars who chose to view their profile. SL also contained other specialized venues for displaying photos publicly. For example, Sara, a 24-year-old wife and mother in RL, used to run a modeling agency in SL that produced a quarterly magazine. Sara would capture images of SL avatar models and distribute them throughout SL in her magazine, which was sold to residents. 


\section{Collocated Photo Sharing}

We also found that sharing was done in a collocated fashion where users in the same area viewed photos on a common display. Photos were dragged and dropped from one's inventory onto editable objects as textures. Objects in SL are editable if one has "modify" permissions for them. In this way, virtually any surface in SL (e.g., walls, cubes, etc.) can instantly turn into a photo sharing display. For example, Figure 3 contains Mike showing us one of his SL wedding photos. The photo is displayed on a large wall near Mike's club. He had modify permissions over the wall and was able to drag the wedding photo out of his inventory and "drop" it on to the wall object. This instantly turned the wall into a photo display. Objects can also be pre-created in SL and scripted to permit collocated sharing; however, this is rarer. For example, two Lifers had "TVs" that could be used in this way; each was scripted to automatically cycle through photos. This is beneficial though we do not know of any tools in SL that incorporate the rich social practices that people often use when sharing photos in RL, for example, annotations [18] and gestures [6].

Collocated sharing was less prevalent than distributed sharing. Only five Lifers engaged in collocated sharing and the frequency of this was much less than distributed sharing. For example, Taylor commonly shared photos with remote SL friends, but saw value in collocated sharing:

$\mathrm{T}$ : often, I'll recieve photos from friends who found

something funny or weird, with the simple message "Hey

look at this"

T: I find that usually, they're just sent, though I love

th idea of getting together and possibly putting them up

on a bulletin board or something

R: but this isn't something you've done yet?

$\mathbf{T}$ : Some. I used to have a bulletin oar in my home and put

photos up on it, and people would come by and ask about

the, .

T: but usually, its just done so quick. A "just happened"

kind of thing

$\mathrm{T}$ : so they snap a photo and send.

$\mathbf{T}$ : like the camera phone thing :)

R: ah okay, so pretty spontaneous

T: quite.

$\mathbf{R}$ : what about the bulletin board type idea appeals to you?

T: I just enjoy anything that makes thing more funand realistic in SL. That, and there's less sending photos aroud and filling up others' inventories. It's accessible by anyone.

In RL, photos are also shared via distributed [11,16,24] and collocated sharing $[6,11,24]$. However, given the widespread possibilities for collocated sharing in SL-any surface can instantly become a display and people can easily teleport to the same location-it is surprising that people are not compelled to share photos more in this manner. That is, even though the VW has less physical and social constraints than RL, people did not necessarily take advantage of it. This likely occurs because distributed sharing is easy to discover and easy to accomplish. For example, the user interface of SL is setup such that distributed sharing options are immediately available after capturing photos. After capture, it is not difficult to drag photos between one's inventory and another avatar to share.

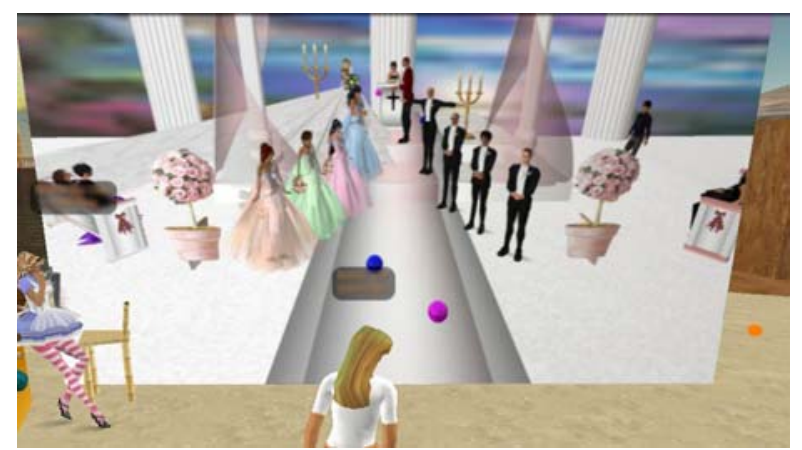

Figure 3. Viewing a photo of a wedding in Second Life.

In contrast, understanding that you can use objects as collocated sharing displays is less obvious because it requires that you understand for which objects you have "modify" permissions. Few people had dedicated collocated sharing devices (e.g., large displays, TVs) and they may not have realized that such devices are not needed. Thus, there are discoverability issues with collocated photo sharing. Because of these issues and overhead, distributed sharing was likely "good enough" to fulfill the photo sharing needs of users. This is not to say that collocated photo sharing applications do not have promise or their place in VWs. They are simply not yet designed in a form that makes them easy to discover and use.

\section{Sharing Photos between the Virtual World and Real Life}

We also probed SL users about sharing photos between RL and SL. Only five of our 20 participants did this and they were all Lifers. For these people, sharing between RL and SL was highly selective. For example, Sara used to display RL photos of her family in her SL office to remind her of her RL. While seemingly a public place, who she invited into her office was a filter on who could view her photos:

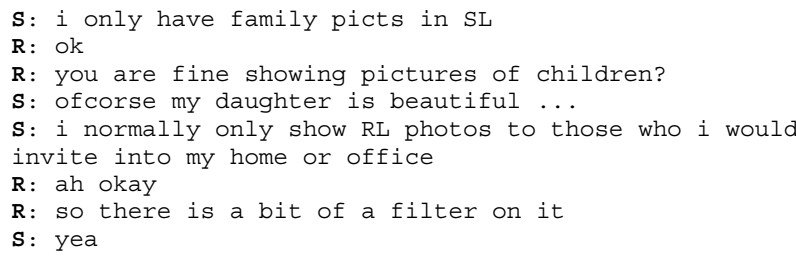

Maggie (40 years old in RL) came to SL to experience her fantasies and participated in a number of science fiction role plays within SL. Here users create their own character and act out a scene spontaneously. Maggie did not want people in SL to know what she looked like in RL, yet she had grown to trust her SL housemates:

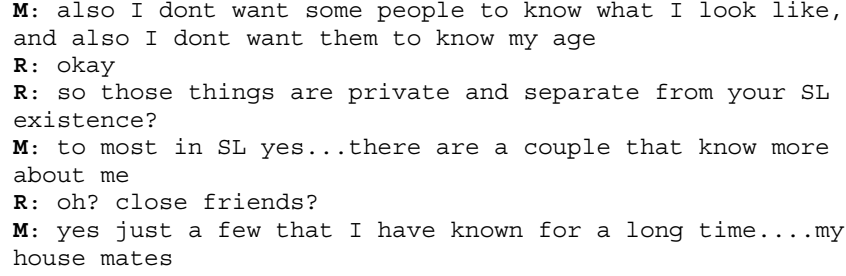

There also existed examples of public sharing of RL photos in SL, although we only encountered two people who did this. For example, Mike had a RL photo of himself in his SL 
profile, which is publicly viewable in SL. For Mike, this was not an issue as his SL was also his RL; the two are blended into one. On the other hand, Sara liked to maintain some degree of RL privacy in SL. Yet she had posted her RL photo-containing her and her RL husband-in her profile in order to dissuade men who only wanted romance from socializing with her.

The opposite-sharing SL photos with people in RL-is also rare (only two Lifers did). For example, Lady shared photos of her SL experiences with a small group of RL work colleagues to "make them smile." Maggie told us that the captain of the ship in her role plays often publicly blogged photos of their SL adventures on the web. Similarly, one can also find hundreds of thousands of SL photos in online sharing spaces like Flickr.

The remaining participants told us that sharing photos between RL and SL is strongly undesired. In Helena's case, her RL family and friends did not know about her SL activities and sharing photos between RL and SL would threaten this secrecy. Others, like Yaleen (35 years old, married in RL), wanted SL to remain a fantasy and bridging it with RL would ruin this:

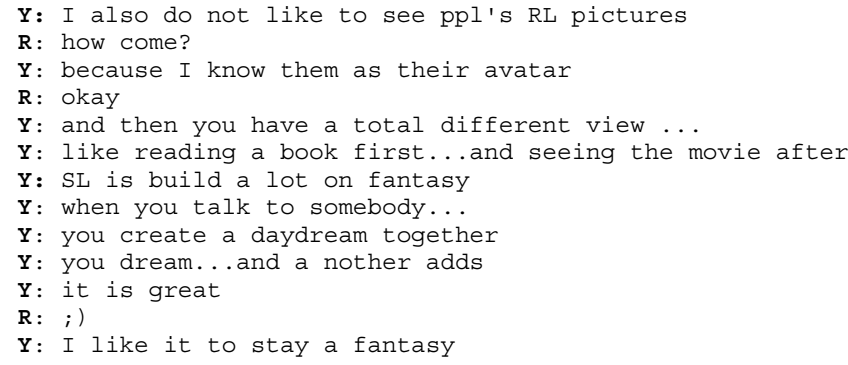

Taken together, we can see that sharing photos between RL and SL was highly selective and in many cases undesired. This presents several challenges. First, it can sometimes be difficult to selectively present photos to only certain individuals in SL. This was the case for Sara who wanted to display photos in her SL office for viewing only by certain people; a careful social protocol was needed to mediate her privacy. Second, challenges exist for those who want their SL activities to remain a fantasy. Those who publicly display their RL photos in SL can easily comprise the need of others for SL to remain a fantasy. Third, some wanted their SL activities to remain private from people in RL. If photos of SL activities are stored locally on one's computer, people in RL could inadvertently or even purposefully come across them.

\section{Diary Keeping and Chat Logs}

We also interviewed a small number of people who kept diaries of their experiences or chat logs. For example, Lady was a 39-year-old teacher, mother of two, and recently separated in RL. She typically spent several hours a day in SL and was planning her virtual wedding at the time we interviewed her. Lady did not take a large amount of photos. Instead she recorded notes in a notebook in RL, much like a diary, to capture her experiences. Some of her SL friends knew she did this and were somewhat fearful:

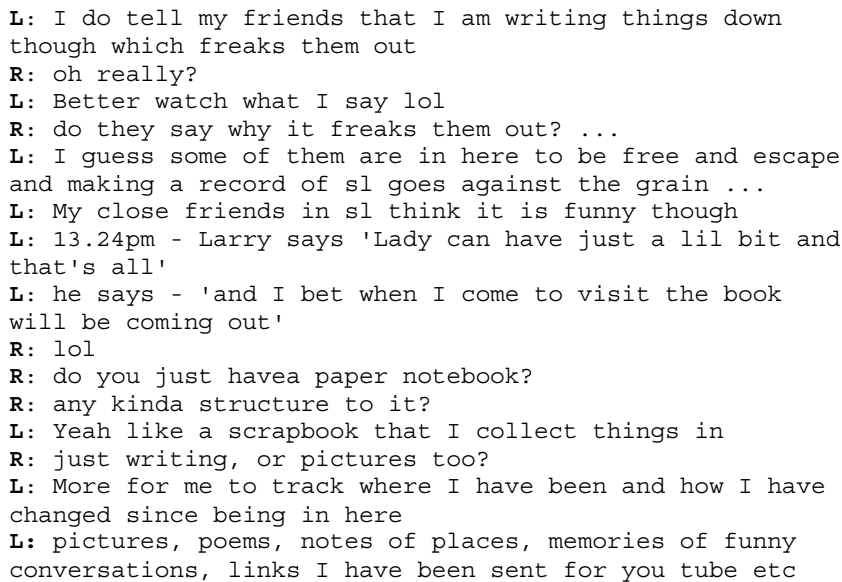

The idea of recording experiences using a diary is similar to work done in the performing arts where narrative captures the rich experiences of VW users [8].

We also had 2 Lifers who kept conversation logs to capture their experiences and also share them with others. For example, Maggie often kept chat logs of her role plays because the conversation was the most important aspect of the experience. The captain of her science fiction role plays also publicly blogged conversation logs from the role plays. Taylor once logged conversations with her new boyfriend as a way to remember the beginnings of their relationship:

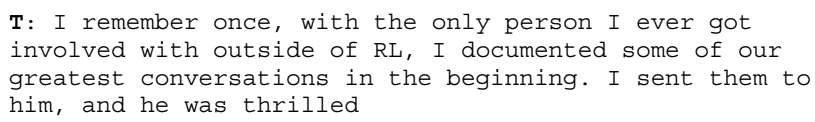

Many IM clients in RL log chats either by default or after setting an option. In fact, these logs are sometimes highly desired for capturing details of one's experiences [14]. However, when one is not at their computer, the type of continuous conversation capture that we saw in SL is much more difficult, if not impossible, to capture in RL.

The Limits of Capturing and Sharing Experiences

We found that a small number of Lifers were less concerned with capturing and sharing their experiences than others. One of the main reasons that this occurred was because some users were more interested in creating new experiences rather than reminiscing about or sharing past ones. Because the VW is not constrained by the physical limitations of RL, it is easy to return to places that you have previously been or meet up with people you have met or interacted with in the past. This makes it easy to reconnect with people and create new experiences with them. It also contrasts RL where long distance travel can be tedious or social obligations (e.g., work) restrict people from reconnecting with family and friends [23].

We also met one Lifer who was not interested in capturing his SL experiences simply because they were not important to him. Mark played music at various clubs in SL and felt 
he was a SL celebrity. As a result, he focused more on providing experiences that others could remember:

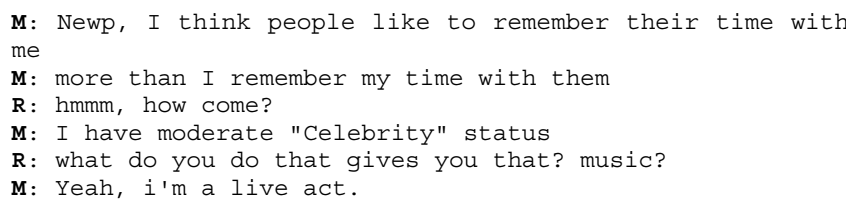

We also probed participants about the use of videos to capture experiences. SL currently has a built-in tool that allows users to capture videos of all actions occurring in one's SL window (e.g., avatar movement, chat, dialog box usage). Yet none of our interviewees recorded videos and many did not even know this feature existed. Maggie told us that even if a video captured an experience, "the excitement of the moment would not be there." This again indicates a desire to experience new situations.

Studies of RL video usage have shown that people typically capture fewer videos than they do photos [16]. This is generally because videos do not serve as better memory triggers than images and they carry the "baggage" of requiring large amounts of time to edit [12]. This may also explain the lack of video usage in SL; however, this explanation seems to contradict the recent proliferation of video capture and online sharing on sites like YouTube. One would expect that the popularity of video in RL would extend to the VW. It also contrasts research on the machinima (machine cinema) subculture, present since the mid 1990s, where users create elaborate animations through the use of computer games or VWs [17,25]. It is likely then that our interviewees simply did not fall within this type of subculture where videos were seen as important. This could also reflect the fact that current interaction between characters in VWs is still somewhat limited, e.g., it is difficult to perform complex actions spontaneously without previously scripting them [21]. Thus, videos of one's actions may not really be warranted for some users and photos sufficed for the types of memory capturing our interviewees performed.

\section{CASUALS}

We now turn to our second set of SL users, whom we call Casuals. These are SL users who were not significantly immersed in the VW and did not have a high degree of permanence. They came to SL on a casual basis, met and interacted with people, and formed relationships (both strong and weak). Yet they did not have a home, partner, or job. Thus, beyond their friendships, they did not have any other social obligations that required them to return to the VW on a continual basis (though many still did).

For example, we interviewed, Larry, a 26-year-old university student in RL who had been coming to SL for four months. Larry felt he was too old to hang out in clubs in RL and so SL was the next best thing to him. Larry liked SL because you could talk to people without social barriers (e.g., being shy) and he enjoyed meeting people that had similar interests as he did. Larry had many friends in SL (even some close ones), but he did not have a home, partner, or job. In fact, he called himself a SL "bum" because he visited a variety of clubs with no real home. Larry's SL experiences mostly involved meeting people and talking with them; thus, he was often concerned with remembering whom he had met and what they had talked about. In addition to Larry, we interviewed other Casuals in SL and some were even less immersed.

\section{Capturing and Sharing Experiences}

Casuals were also interested in capturing and sharing their experiences though to a much less extent than Lifers. In fact, only 3 of 8 Casuals in our study reported capturing and sharing their SL experiences. Like Lifers, these routines typically involved the use of photos. Photo capture by Casuals was highly selective though and they typically had only a few photos (less than 10). For example, Koho was 48 years old in RL and came to SL to meet women. He told us that he only took photos of very beautiful avatars. In his 11 months in SL, this had amounted to only five photos. Casuals who captured experiences through photos only shared their photos with others via distributed sharing. The amount of sharing was much less than Lifers though, considering that fewer photos were taken. We did not find any instances of collocated photo sharing for Casuals.

The five Casuals from our study who did not capture their experiences using photos simply did not know how to take photos, or were skeptical of how valuable photos would be even if they knew how to capture them. For example, Antwone (47 years old in RL) had been coming to SL for less than one month. He commented on using photos:

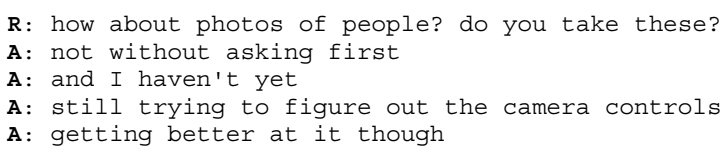

We also saw one Casual who relied on conversation logs to capture her experiences. This was because she had two avatars and needed to remember the activities of each:

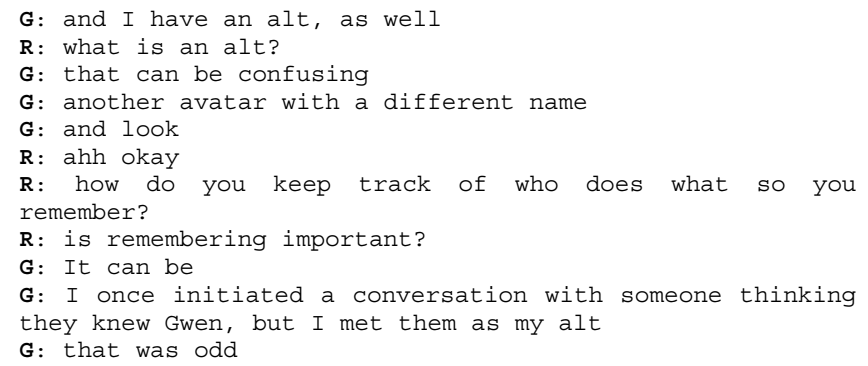

Martin is an example of a Casual who was not interested in capturing experiences at all. Martin only came to one place in SL, although he engaged in conversations with new people all the time. Despite this, he did not collect photos or videos and was not interested in establishing a SL identity.

Taken together, we can see that Casuals captured and shared experiences to a much lesser extent than Lifers. Yet our data revealed that there was more to memories than 
simply experiences. Many Casuals were highly interested in recording facts about their SL existence. This was actually a phenomenon that encompassed both Lifers and Casuals.

\section{RECORDING AND (SOMETIMES) SHARING FACTS}

All of the participants in our study had one or more techniques that they employed to capture or record facts about their SL existence. By facts we are referring to specific details about people or places, rather than actual experiences that occurred with a person or in a particular location. The recording of facts was popular amongst all users (Casuals and Lifers); however, the sharing of facts was more restrictive and only happened in certain cases.

\section{Keeping Track of Who You Know}

All participants in our study kept track of people who they had met in SL, albeit some more than others. The intent was to remember contacts and details about them and also establish ways to reconnect with them at future points in time. All interviewees did this through a Friends List contained in the SL interface. The list is private to each user and looks very similar to contact lists found in instant messaging (IM) clients, showing who is online vs. offline. Users request friendship from one another and, if accepted, both parties add the person's name to their list. From the Friends Lists, users can view a person's profile, chat with the person, or teleport to their location.

Typical usage of the Friends List involved keeping a general list of contacts where people need not share a close relationship. Some lists grew large because people were trying to meet others and establish relationships. Over time, many users pruned their lists though.

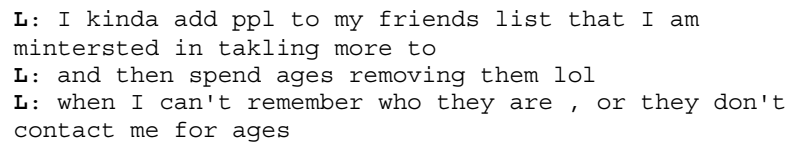

Sometimes contact lists became more exclusive and there were several levels of friendships. For example, Yaleen had 15 friends on her contact list, of which she said half were "special" friends. The challenge here is that all friends are kept within the same list as SL does not currently permit the grouping of contacts according to varying degrees or levels of friendship [23]. This made it difficult to find close contacts who one may have wanted to repeatedly contact.

Being on one's contact list also had social implications. The "offering of friendship"-requesting to add someone to your Friends List-was often timely. That is, some people found it rude to ask too soon and others felt they should wait for the other person to ask first (e.g., a male waiting for a female). The removal of a person from the Friends List sometimes caused distress as contact lists are reciprocal and this visible act could be noticed. Taylor commented:

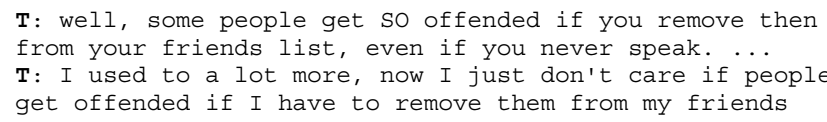

T: If I wouldn't mind running into them again, I offer friendship anyway :)

Taylor had also started using contact cards as a form of "Friends List Lite" to avert negative social feelings. These were like business cards that were shared and stored in one's inventory. Taylor would initially give these out rather than offering friendship to others. IM contact lists in RL have also been found to carry social implications with them. For example, teenagers have been found to use the size of one's contact list as a popularity indicator [13].

Some SL users also explicitly recorded notes about the people with whom they interacted (2 Lifers, 3 Casuals). SL contains a feature called Notes that lets you add notes to each avatar's profile in a free-form textbox. In contrast to the rest of the avatar's profile, these notes are only viewable by the person who added them; they are neither publicly viewable nor viewable by the person whose profile contains them. This means that each time you see a person, you can open his/her profile and view your notes about him/her. For example, Maxwell (32 years old and a Casual) was most interested in meeting new people and conversing with them. Because of this, he used the Notes feature to record basic information about people such as their age, origin, and likes/dislikes.

The important thing when it comes to note taking is the privacy of notes: they were never shared with others. Users were quite adamant about this because they may record information that they did not want others to see. Yet the challenge with Notes in SL is a mismatch between the user's conceptual model of how Notes work and the model used in the user interface. Notes are private, but kept within another user's profile, which is by default public. This mismatch made it difficult for many interviewees to fully trust that their comments would not be seen by others. Because of this, some users saved notes outside of the SL user interface as chat logs or in text files.

In RL, many people capture notes in the workplace using scrap pieces of paper, a notebook, or computer files [14]. However, these notes are often less focused on individuals and more on facts people need to remember about their activities. Of course, there are no RL facilities that provide a notebook attached to each person that we encounter. PDAs could serve this purpose but rarely are used in this way. Even if there was a way to record information about each person in RL, there would likely be immense social pressure to not perform such acts as information you write about another is at risk to become public. Thus, the VW permits a practice that is not easily replicated in RL because of lack of physical and social constraints.

\section{Keeping Track of Where You've Been}

Nearly all participants in our study kept track of a subset of the places that they had been in SL. The dominant means to do this was with Landmarks (denoted as LMs in SL chat): a hierarchical list of locations (much like web bookmarks [1]). Users added to this list in their inventory and used them as instant teleportation destinations. As with photos, 
users could drag and drop LMs from their inventory onto other avatars, who would then receive the LM. Upon receipt, they could teleport to the LM or store it in their own inventory. This was the primary mechanism for sharing location information in SL, which we found to be a common practice. For example, newcomers to SL would often receive LMs from more experienced users who showed them good places to shop or visit. As with photos, the organization of LMs in one's inventory could be a challenge. Some, like Gwen, took the time to group LMs according to location types (e.g., shopping, dancing). However, there were others who had a large set of LMs that remained ungrouped and difficult to search through.

\section{DISCUSSION AND REFLECTION}

Our study has generated a descriptive account of the ways in which people capture and share memories in the VW. Many people had experiences in SL that were very much like RL. These ranged from casually meeting and interacting with others (Casuals) to creating lifelike situations involving partners, jobs, or residences (Lifers). Lifers were focused on capturing and sharing both experiences and facts about their SL activities. Casuals were focused mostly on capturing facts with a lesser need to record experiences.

To fulfill these needs, Lifers and Casuals used a range of tools. In certain circumstances, the tools that people were provided with in SL mapped too well to what people do in RL. This precluded people from breaking the physical and social bounds of RL and finding new ways to capture or share their virtual experiences. Instead, they ended up replicating what they did in RL. This occurred for photo usage. Essentially the photo capture and sharing routines that people used in SL were (nearly) the same as they are in RL. One can seemingly capture a photo from any angle or zoom level in SL, yet this was not done. People constructed the same type of posed portraits that they do in RL and captured the same spontaneous moments. When it came to sharing photos, people did not typically take advantage of the large range of photo display possibilities (e.g., any surface could become a collocated photo display). Other tools like conversation logs, notes, friend lists, landmarks, or teleporting offered unique opportunities to capture and share memories in ways not easily possible in RL.

Replicating RL is certainly not bad. In some situations, it may even be desirable because it allows people to transfer their knowledge and understanding from the real world into the VW. Yet replicating RL routines and tools does not do the VW justice. The VW holds many design possibilities that are not bound by RL constraints. Any situation, context, or experience can theoretically be (re)created in the VW. One could imagine leveraging this with memory tools. For example, photo viewing applications could link photos with the location where they were captured and the people who were there. Tools could allow people to then return to this location with the same people, or even view photos in their original context. People could even relive another person's experiences. Thus, we argue that designers should move beyond what is possible in RL and design tools that take advantage of the unconstrained nature of VWs.

We can also use the VW to learn about RL. That is, we can observe what people do in VWs where they are unconstrained by the physical and social constraints of RL and imagine the applications of this in RL. Thus, VW behaviors and routines can act as a catalyst for RL technology designs that step beyond everyday practice. For example, people could create photo displays out of most objects in the VW. Yet in RL this is not currently possible. However, imagine portable devices (e.g., mobile phones, digital cameras) that could transform any surface into a photo sharing display through projection technologies (such as those found in [26]). Rather than having a group huddle around a mobile phone display to look at a photo [16], the mobile phone could display the photo on the nearest wall at a larger size.

Of course, there are caveats to this general design approach. A straight crossover of VW designs into RL is not always appropriate. Designers must consider people's RL routines and the impact that design decisions would make on them. For example, conversations can be easily logged in VWs and, in fact, we saw that for some people this was a highly valuable tool. However, imagine the same tool in RL where people could record all voice conversations that occur throughout a given day. This tool could be very valuable for remembering facts, but would naturally be intrusive to the privacy of others nearby, and possibly even to the main user. Recording selective conversations could be more valuable, especially in situations where people suffer from memory loss. Other designs may also pose similar social and cultural issues and should be considered in this manner.

Hindmarsh et al [15] also suggest that we can learn about RL from the VW. Yet they explore this idea in the context of VWs for workplace collaboration, not VWs aimed at social activities. Moreover, they suggest learning about RL based on the constraints found in VWs, rather than our suggestion of the opposite (a lack of constraints).

\section{CONCLUSION}

We have studied the social culture of SL through in-world interviews with SL users. Here we uncovered two categories of user behaviors for capturing and sharing memories (Casual/Lifers) focused on the use of photos, diaries, landmarks, friend lists, and conversation logs. This builds on previous work that highlights the use of blogs [29], narrative [8] and videos [17,25] for recording and sharing VW experiences. We also showed how the lack of physical and social constraints in the VW affects user routines and, in some cases, how it does not. This knowledge can and should be used as a catalyst for creative design ideas that can be applied to either the VW or RL. These findings are not present in the collaborative virtual environment (CVE) literature as many studies over the past decade have been focused on VWs aimed at workplace collaboration where people know each other in RL, as 
opposed to the type of environment we studied. Even still, we do not know of any studies that have looked specifically at memory capture and sharing in MUVEs focused on social activities.

We have studied one specific VW, yet it is likely that our results are broadly applicable to other VWs of the same genre given that they are all focused on the same goal: users aim to socialize and meet others. Thus, it is likely that people will generate similar experiences and have similar desires to capture and share memories. The tools that people use to capture and share memories will be limited to what the VW provides however.

\section{ACKNOWLEDGMENTS}

Special thanks to Tim Nichols, Jeff Snyder, Deniz Schildkraut, Dave Barnum, Joel Lawther, and Rodney Miller. We are also indebted to our gracious participants.

\section{REFERENCES}

1. Abrams, D., Baecker, R., and Chignell, M. Information Archiving with Bookmarks, Proc CHI 1998, ACM Press (1998).

2. Bardzell, S., and Bardzell, J. Sex-Interface-Aesthetics: The Docile Avatars and Embodied Pixels of Second Life BDSM, Proc. CHI 2006, ACM Press (2006).

3. Bartle, R. Designing Virtual Worlds, New Riders Publishing (2004).

4. Brown, B., and Bell, M. CSCW at Play: 'There' as a CVE, Proc. CSCW 2004, ACM Press (2004).

5. Chalfen, R. (1987), Snapshot Versions of Life, Bowling Green State University, Ohio, Popular Press.

6. Crabtree, A., Rodden, T., and Mariani, J. Collaborating around Collections, Proc CHI 2004, ACM Press (2004).

7. Dourish, P. Introduction: The State of Play, JCSCW Special Issue: Interaction and Collaboration in MUDs, 7(1-2), Springer, Netherlands (1998).

8. Doyle, D., and Kim, T. Embodied narrative: the virtual nomad and the meta dreamer, International Journal of Performance Arts and Digital Media, 3: 2\&3, (2007).

9. Ducheneaut, N., Yee, N., Nickell, E., and Moore, R. “Alone Together?” Exploring the Social Dynamics of MMOG, Proc. CHI 2006, ACM Press (2006).

10. Evard, R., Churchill, E., and Bly, S. Waterfall Glen: Social Virtual Reality at Work, In Collaborative Virtual Environments, Springer Verlag (2001).

11. Frohlich, D., Kuchinsky, A., Pering, C., Don, A., and Ariss, S. Requirements for Photoware, Proc. CSCW '02, ACM Press (2002), 166-175.

12. Frohlich, D., and Fennell, J. Sound, Paper and Memorabilia, Personal \& Ubiquitous Computing, 11, (2007) 107-116.

13. Grinter, R., Palen, L., and Eldridge, M. Chatting with Teenagers: Considering the Place of Chat Technologies in Teen Life, ACM ToCHI (2006), 423-447.
14. Halverson, C. The Value of Persistence: A Study of the Creation, Ordering and Use of Conversation Analysis, Proc. HICSS 2004, IEEE (2004).

15. Hindmarsh, J., Fraser, M., Heath, C., Benford, S., and Greenhalgh, C. (1998) Fragmented Interaction: Establishing mutual orientation in virtual environments, Proc. CSCW 1998, ACM Press.

16. Kindberg, T., Spasojevic, M., Fleck, R., and Sellen, A. The Ubiquitous Camera, IEEE Pervasive Computing: Mobile and Ubiquitous Systems, 4(2), IEEE Computer Society (2005), 42-50.

17. Lowood, H. High-Performance Play: The Making of Machinima. Videogames and Art: Intersections and Interactions, Intellect Books (2005).

18. Makela, A., Giller, V., Tscheligi, M., and Sefelin, R. Joking, Storytelling, Artsharing, Expressing Affection, Proc. CHI 2000, ACM Press (2000), 548-554.

19. Maslow, A. A Theory of Human Motivation, Psychological Review, 50 (1943), 370-96.

20. Miller, A., and Edwards, K. ,Give and Take: A Study of Consumer Photo-Sharing Culture and Practice, Proc. CHI 2007, ACM Press (2007), 347-356.

21. Moore, R.J., Ducheneaut, N., and Nickell, E. Doing Virtually Nothing: Awareness and Accountability in Massively Multiplayer Online Worlds, JCSCW (2006).

22. Nardi, B., Whittaker, S., and Bradner, E. Interaction and Outeraction: Instant Messaging in Action, Proc. CSCW 2000, ACM Press (2000), 1-10.

23. Neustaedter, C., Elliot, K., and Greenberg, S. Interpersonal Awareness in the Domestic Realm, Proc. OzCHI, (2006).

24. Nunes, M., Greenberg, S., and Neustaedter, C., Sharing Digital Photographs in the Home through Physical Mementos, Souvenirs, and Keepsakes, Proc. DIS 2008, ACM Press (2008).

25. Picard, M., Machinima: Video Game as an Art Form? Proc. CGSA 2006, (2006).

26. Pinhanez, C. The Everywhere Displays Project: A Device to Create Ubiquitous Graphical Interfaces, Proc. Ubicomp 2001, Springer-Verlag (2001).

27. Rodden, K., and Wood, K. R. How do People Manage Their Digital Photographs?, Proc. CHI 2003, ACM Press (2003), 409-416.

28. Strauss, A., and Corbin, J. Basics of Qualitative Research, SAGE Publications (1998).

29. Walker Rettberg, J. Blogging: Digital Media and Society, Polity Press (2008).

30. Waskul, D., and Douglas, M. Cyberself. The Emergence of Self in On-Line Chat, The Information Society, 13, (1997) 375-397.

\section{CREDITS}

Second Life is a trademark of Linden Research, Inc. Certain materials have been reproduced with the permission of Linden Research, Inc. COPYRIGHT (C) $2001-$ 2008 LINDEN RESEARCH, INC. ALL RIGHTS RESERVED. 